\title{
Organosole von Metallen und Metallhyden der Platingruppe III.
}

\author{
Von Con rad Amberger (Erlangen). (Eingegangen an 30. Angust 1915,)
}

In zwei Mitteilungen' habe ich über die Darstellung von kolloidem Platin und Palladium und deren Hydroxyden berichtet; sie wurden als Adsorptionsverbindungen mit Wollfett in Form von Organosolen gewonnen. Als Reduktionsmittel zur Herstellung der elementaren Metalle diente Hydrazinhydrat. Die Beimengung von Wollfett verlieh diesen Kolloiden große Beständigkeit, so daB sie in salbenartigen und auch in fester Form jahrelang haltbar sind und in organischen Lösungsmitteln sich auch nach jahrelanger Aufbewahrung noch 10̄sen. Im Anschluf an diese erwähnten Untersuchungen habe ich nummehr auch kolloides $\mathrm{Osmium}$ und eines seiner Hydroxyde dargestellt.

Versuche zur Darstellung von kolloiden Osmiumlösungen in Form von Hydrosolen sind vor einer Reihe von Jahren schon von $N$. Castorol), sowie von $A . G u t b i e r$ und $K$. Hof mei e ${ }^{3}$ ) getnacht worden. Ersterer führte an Schlusse seiner Mitteilungen an, daß er das Osmiumhydrosol unter Anwendung von Akrolein als Reduktionsmittel erhalten habe. In Gemeinschaft mit dem Verfasser vorstehender Arbeit ist von $\mathrm{C} . \mathrm{Pa}$ a ${ }^{4}$ ) gezeigt worden, dab Aldehyde, wie z. B. Formaldehyd, Osmiumlösungen nur bis zum Osmiumhydroxyd reduzieren. $\mathrm{DaB}$ durch Akrolein eine weitergehende Reduktion als durch Formaldehyd erfolgen sollte, ist nicht anzurehmen, somit war auch das nach dem Castoro'schen Verfahren hergestellte Kolloid kein Osmiumhydrosol, sondern Osmiumdioxyd in wasserlöslicher form. Gutbier und Hof me i e r reduzierten Kaliumosmiatlösung mit Hydrazinhydrat bei Gegenwart von arabischem Gumni als Schutzkolloid. Sie erhielten ein Hydrosol in starker Verdünnung; aber auch nicht elementares Osmium, sondern eine Osmiumsauerstoffverbindung mit etwa 4,5 Proz. Sauerstoff.

Ein wirkliches Osniumhydrosol in fester Form von großer Beständigkeit erhielten C. Paal und C. Amberger ${ }^{5}$ ), indem sie Alkaliosmiat bei

1) C. Amberger. Koll. Zeitschr. 13, 310-313 und $313-318$.

i) N. Castoro, Zeitschr. f. anorg. Chem. 41, 126.

s) A. Gutbier und K. Hofmeier, Journ. f. prakt. Chem. (NF) 11,456 .

t) C. Pa a I, Ber. d. Deutsch. chem. Ges. 40, 1378 bis 1391 (1907).

b) C. Paa! und C. Amberger, Ber. d. Deutsch. chem. Ges. 40, 1392-140.\& (1907).
Gegenwart von protalbin- bzw. lysalbinsaurem Natrium zunächst mit Hydrazinhydrat reduzierten. Dadurch wurde eine Osmiumsauerstoffverbindung in kolloider Form gewonnen. Wurde das so erhaltene Produkt in gepulvertem $\mathrm{Zu}$. stande in einer Wasserstoffatmosphäre auf $30-40^{\circ}$ erwärmt, so wurde es vollständig zu eiementarem Osmium reduziert, das sich in Wasser leicht mit den, dem kolloiden Osmium charakteristischen Eigenschaften löste.

Ueber die Darstellung von kolloidem Osmium in festem, haltbaren Zustande in Form eines Orga no sols ist in der Literatur noch nicht berichtet worden. Die Darstellung von Osmiumorganosolen gelingt nach im folgenden mitgeteiltem Verfahren:

Kolloide Osmiumpralparate werden in ähnlicher Weise erhalten wie die von mir beschriebenen Organosole des Platins und Palladiums. Wollfett wurde mit Alkaliosmiatlösung verrieben und die Mischung reduziert. Lost man. diese Präparate in Petroläther, so lassen sich durch Fällen mit Alkohol Produkte mit höherem Osmiumgehalt erhalten. Als Reduktionsmittel diente zunächst Hydrazinhydrat. Das durch Hydrazinhydrat reduzierte Produkt enthält aber nicht kolloides Osmium, sondern entweder Osmiumdioxyd oder eine Mischung von 1) smium und einer Osmiumsauerstoffverbindung. Denn in der Arbeit von Paal und Amberger ${ }^{6}$ ) ist nachgewiesen worden, daß alkalische Osmiatlösungen durch Hydrazinhydrat nicht $z$ in elementarem Osmiuin reduziert werden, und dab auch die Einwirkung von Hydrazinhydrat auf derartige Osmiatiôsungen keineswegs zur quantitativen Abscheidung führt. Aus dem analytischen Material dieser Arbeit: "Zur Kenntnis des Osmiums " ist ersichtlich, dab bei der Reduktion von Osmiaten in Gegenwart von überschüssigem Alkali durch Hydrazinhydrat ein Osmiumoxyd bzw. ein Osmiumoxydhydrat zur Abscheidung gelangt, dessen Sauerstoffgehalt dem des Osmiumdioxydes nahe kommt. Wirkt dagegen Hydrazinhydrat nur in schwach alkalischer Lösung auf Kaliumosmiatlösungen, so enthält das Reduktionsprodukt nur noch 2,7 bis 3 Proz. Sauerstoff.

6) C. Paal und C. Amberger, Ber. d. Deutsch. chem. Ges. 41, $1378-1391$ (1907). 
Bei meinen Versuchen wirkte Hydrazinhydrat auf eine von Wollfett adsorbierte, stark alkalische Osmiatlösung ein, somit war das Reduktionsprodukt nicht Osmium, sondern Osmiumdioxydhydrat. Dieses primäre Reduktionsprodukt in Adhäsion mit Wollfett löste sich in Petrolather und kann aus dieser Lösung durch Alkohol gefält werden. Bei einer bestimmten Konzeniration werden nun nicht mehr salbenartige Produkte gefallit, sondern solche von fester Konsistenz. Werden derartige feste Fallungsprodukte in zerkleinerter, pulveriger Form bei einer Temperatur von $50-60^{\circ}$ in einer Wasserstoffatmosphäre erwärmt, so werden sie reduziert und man erhält Organosole von elementarem Osmium, die sich auch nach der Reduktion noch leicht und vollständig in Aether, Petrolăther, Benzin, Benzol, fetten Oelen und dergleichen lösen. Im Gegensatz zu den Hydrosolen des Osmiums oxydieren sich diese Präparate weniger leicht. Wird kolloides, wasserlösliches Osmium in festem Zustand einige Zeit im Exsikkator aufbewahrt, so macht sich der charakteristische Geruch nach Osmiumtetroxyd bemerkbar und die Substanz verliert stetig an Gewicht. Dagegen konnte bei diesen Präparaten auck bei längerer. Aufbewahrung der Geruch nach Osmiumtetroxyd nicht wahrgenommen werden und Substanzverlust war nicht nachweisbar. Ebenso lassen sich, wie aus den Adsorptionsverbindungen der bereits beschriebenen Organosole, aus den flüssigen Osmiumorganosolen feste Präparate gewinnen, wenn man sie mit Alkohol aus ihren Lösungen fält. Diese Fällungen lösen sich, wenn sie nicht aus $2 u$ stark verdünnten Lösungen vorgenommen werden, wieder mit den ursprünglichen Eigenschaften in den verschiedenen Medien; zeigen dann aber einen höheren Gehalt an Metall, da bei der Fällung Anteile des Schutzkolloides in Lösung bleiben.

\section{Analyse der salbenartigen und festen Osmiumorganosole.}

Bei der Analyse derartiger Osmiumpräparate, die neben Metall Wollfett enthalten, kam es lediglich darauf an, den Osmiumgehalt festzustellen. $\mathrm{Pa}$ al hat in Gemeinschaft mit dem Verfasser vorstehender Abhandlung in der Arbeit "Zur Kenntnis des Osmiums" über verschiedene Verfahren berichtet, nach denen es moglich ist, Osmium aus seinen löslichen Verbindungen quantitativ abzuscheiden und zu bestimmen. Als gecignetste Methode wurde die auf folgender Grundlage beruhende erkannt. Osmium- tetroxyd in wässieriger Kalilauge gelöst, wird durch Alkohol zu Kaliunosmiat reduziert. Reine Osmiatlösungen zerfallen nach Untersuchungen von Fremy und $\mathrm{Claus}$ beim Ansäuern in Osmiumdioxyd und Osmiumtetroxyd. Werden somit derartige Osmiatlösungen mit verdünnter Schwefelsäure angesäuert, so scheidet sich Osmiumdioxyd unlöslich ab und Osmiumtetroxyd bleibt in Lösung. Letzteres wird durch die Gegenwart von Alkohol ebenfalls zu Dioxyd reduziert, so dab es möglich ist, alles Osmium aus reiner alkalischer Osmiatlosung durch Schwefelsăure und Alkohol quantitativ in Form von Osmiumdioxyd abzuscheiden. Der filtrierte und getrocknete Niederschlag wird dann im Wasserstoffstrom zu elementarem Osmium reduziert und gewogen.

Die in Betracht kommenden Präparate waren nun in Verbindung mit Wollfett gewonnen worden; es war für die Analyse notwendig, das Osmium von dem Wollfett zu trennen, um dieses dann in Natrium- oder Kalium-Osmiat überführen zu können.

Vor einer Reibe von Jahren hat G.v.Knorre ?) zur Bestimmung des Kohlenstoffs neben Osmium in osmiumhaltigen Kohlenfäden ein Verfahren angegeben, das auf der Verbrennung beider Elemente im Sauerstoffstrom beruht, die dabei in Osmiumtetroxyd und Kohlensäure übergehen. In ähnlicher Weise wie die im Verein mit $\mathrm{P}$ a a I hergestellten Hydrosole wurden auch die unter Verwendung von Wolliett als Schutzkolloid gewonnenen Organosole im Sauerstoffstrom verbrannt und die Verbrennungsprodukte in wässerigem Alkali, dem etwas Alkohol zugesetzt war, aufgefangen. Das entstehende Osmiumtetroxyd wird in die Alkalilösung übergeführt, und diese dann durch Schwefelsäure gefallt.

Die Ausführung der Analyse geschah in folgender Weise:

Die Substanz wurde in einem Porzellanschiffchen abgewogen, dieses in eine Verbrennungsröhre gebracht und das Rohr auf einem Verbrennungsofen erhitzt. Das eine Ende des Rohres war nach abwärts gebogen und zu einer engen Röhre ausgezogen, die mit zwei, die Adsorptionsflüssigkeit enthaltenden $U$ förmigen Kugelröhren in Verbindung stand. Das ausgeżogene Ende des Verbrennungsrohres taucht weit in das erste $U$-Rohr bis etwa unter die Oberflăche der Adsorptionsflüssigkeit ein. Die Verbrennung geschah in einem Strom von

7) G. v. Knorre, Zeitsch. f. angew. Chem 15 , 393 (1902). 
reinem, trockenen Sauerstoff und wurde so geleitet, daB das Wollfett nahezu vollständig zu Kohlensäure verbrannte; bei einiger Vorsicht ging der Verbrennungsproze $B$ glatter vor sich als beim Verbrennen der festen Hydrosole. Nachdem alles Osmiumtetroxyd in die U-Rôhre übergetrieben war, wurde die Adsorptionsflüssigkeit schwach erwărmt. Als Adsorptionsflüssigkeit diente verdünnte alkoholhaltige Natriumlauge. Die violettgefärbte Natriumosmiatlösung wird aus dem Adsorptionsapparat in ein Becherglas übergeführt und mit verdünnter Schwefelsäure angesäuert. Nach etwa $10-12$ stündigem Stehen hatte sich der Niederschlag derart abgesetzt, daß die überstehende Flüssigkeit farblos und frei von Osmium war. Die Fällung wurde dann in einem Asbestfiiterröhrchen filtriert. Der Niederschlag wird dann noch mit alkoholhaltigem Wasser bis zum Verschwinden der Schwefelsăurereaktion ausgewaschen. Das Filterrohr wurde dann in einem evakuierten Exsikkator getrocknet. Zur Ueberführung des Oxydhydrates in Osmium wurde das Asbestfilterrohr mittelst eines Gabelrohres mit einem Kohlensäure- und Wasserstoffapparat verbunden, um so durch die Koblensäure den Luftsauerstoff $z u$ verdrängen, so daß ein Erhitzen der Substanz in reiner Wasserstoffatmosphäre möglich war.

1. Darstellung von kolloidem Osmium in Form des Organosols.

Präparat 1: Zwei Teile Osmiumtetroxyd $(=1,48$ Teile Os) werden unter Zusatz von wenig Natronlauge in sieben Teilen Wasser gelöst und diese Lösung durch Zugabe von Alkohol zu Natriumosmiat reduziert. In 13 Teile durch gelindes Erwärmen erweichtes Wollfett wurde die völlig reduzierte Natriumosmiatflüssigkeit in kleinen Anteilen unter stetem innigen Verreiben eingetragen. Ist alles $\mathrm{Na}$ triumosmiat eingetragen und mit Wollfett gleichmäBig verrieben, so gibt man tropfenweise Hydrazinhydrat zu. Beim Vermischen der Salbenmasse mit Hydrazinhydrat färbt sich diese dunkler, bläht sich auf, nimmt metallgrauen Farbenton an; auf weiteren Zusatz von konzentriertem Hydrazinhydrat und tagelangem Stehen geht erst allmählich die graue Farbe in schwarz über; durch Erwärmen und weiteres inniges Verreiben der Masse mit neuen Mengen Hydrazinhydrat wird die Reduktion beschleunigt und beendigt. Die Farbe ist dann schwarzbraun.

Zur Entfernung der Reaktionsnebenprodukte wurde das Präparat zunächst im Vakuum über Schwefelsäure getrocknet. Das trockene Pria- parat wurde in $50 \mathrm{ccm}$ Petroläther gelöst und die Aetherlösung dreimal mit je $100 \mathrm{ccm}$ Wasser ausgeschüttelt. Die atherische Plüssigkeit wurde mit Chlorkalzium getrocknet, von dem Trockenmittel abfiltriert und das Lösungsmittel abdestilliert. Das Präparat bildete eine schwarzbraune, salbenartige Masse, die leicht von organischen Lösungsmitteln wie Petroläther, Chloroform, Tetrachlorkohlenstoff, fetten Oelen und anderen Flüssigkeiten, die Wollfett lösen, aufgenommen wurde. Die Lösungen erscheinen im durchfallenden Lichte klar und zeigen schwarzbraune Farbe. Die Ausbeute betrug $10,8 \mathrm{~g}$.

Für die Analyse wurden $5,4 \mathrm{~g}$ des Präparates in $100 \mathrm{ccm}$ Petroläther gelöst und durch etwas mehr als das gleiche Volumen Alkohol gefällt. Der trockene Fällungsrückstand $=2,1 \mathrm{~g}$ diente zur Bestimmung des Osmiumgehaltes. Die Analyse geschah durch Verbrennen der Substanz im Sauerstoffstrom; das Osmiumtetroxyd wurde durch alkoholhaltiges, wässeriges Alkali adsorbiert; aus dieser Lösung des Osmiumdioxyds durch Schwefelsïure gefällt und der Niederschlag zu Osmium reduziert.

Angew. Subst. 2,0 g, gef. Os 0,408, Proz. Os $=20,4$.

Das durch Alkoholfällung erhaltene Präparat enthält 20,4 Proz. Os. 4,2 $\mathrm{g}$ dieses Präparates entsprechen $10,8 \mathrm{~g}$ des nicht gefällten Präparates. Demnach betrug der Osmiumgehalt des ursprünglichen Präparates 7,9 Proz.

Präparat 2: Nach dem vorstehend beschriebenen Verfahren erhält man ein Präparat, das neben Osmium noch Osmiumsauerstoffverbindungen enthält, da, wie bereits erwähnt, die Reduktion mit Hydrazinhydrat nicht ausschlie日lich zu elementarem Osmium führt. Um zu einem osmiumreicheren Präparat zu gelangen, das nur Osmium in kolloider Form enthäl, wurde in folgender Weise verfahren:

In 13 Teile durch gelindes Erwärmen erweichtes Wollfett wurde eine Lösung von Natriumosmiat eingetragen. Diese wurde erhalten dadurch; dab zwei Teile Osmiumtetroxyd in acht Teilen Wasser unter Zusatz von Natriumhydroxyd gelöst und dirrh einige Tropfen Alkohol reduziert wurden. Zur Salbenmasse wurde dann, wie bereits im Präparat 1 beschrieben, Hydrazinhydrat zugegeben. Nach beendigter Reduktion wird die Salbenmischung durch Erwărmung erweicht und in 140 Teilen Petroläther gelöst. . Die Lösung ist blauschwarz bis schwarzbraun, je nach dem Verlauf des Reduk. tionsprozesses; aus ihr wird durch Zugabe von 
$200 \mathrm{ccm}$ Alkohol nahezu das gesamte Osmium in Adhäsion mit bestimmten Teilen des Wollfettes gefallt. Die Reaktionsprodukte werden in der Weise entfernt, dah man zunächst Lösungsund Fällungsmittel möglichst voin Sediment abgieit und letzteres mit heißem Wasser von $50-60^{\circ}$ digeriert. Dies wird solange wiederholt, bis die Waschflüssigkeit nicht mehr alkalisch reagiert. Der so gereinigte Niederschlag wird, nachdem er so weit wie möglich von Wasser befreit ist, in eine Glasschale gebracht und bei einer Temperatur von 40 bis $50^{\circ}$ in vacuo über Schwefelsäure getrocknet.

Man erhält so eine schwarze amorphe Masse von fester nicht mehr salbenartiger Konsistenz von sonst gleichen Eigenschaften wie Präparat 1. Das Präparat enthäit nicht ausscblieflich Osmium als elementares Kolloid, sondern es ist im wesentlichen das feste Organosol eines Oxydhydrates dieses Elementes in Adsorption mit bestimmten Anteilen des Wollfettes. Um dieses Oxydhydrat in Osmium überzutühren, wird die pulverförmig zerkleinerte Masse mit trockenem Wasserstoff zunächst in der Kälte, dann bei $30-50^{\circ}$ reduziert. Zur Vermeidung von explosivem Knallgas wird die Luft durch Kohlensäure und nach beendigter Reduktion durch dieses Gas der Wasserstoff verdrängt. Das so erhaltene Organosol des Ostniunss zeigt die gleichen Eigenschaften, wie vor der Reduktion, insbesondere ist es noch eben so leicht löslich in den organischen Lösungsmitteln, die Wollfett lösen.

Die Analyse wurde in der wiederholt be. schriebenen Weise ausgeführt. Die Substanz wurde direkt im Sauerstoffstrom verbrannt, das sich bildende Osmiumtetroxyd in Natronlauge bei Gegenwart von Alkohol aufgefangen und aus der Kaliumosmiatlösung durch Schwefelsăure das Dioxyd gefält und im Wasserstoffstrom reduziert:

Angew. Subst. : 1,23 g; gef. Os: 0,258; Proz. Os $=20,97$ Proz.

\section{Darstellung von kolloidem Osmiumdioxyd.}

In Vorstehenden wurde bereits erwähnt, daß aus Kaliumosmiatlösung bei Gegenwart von Ałkohol Osmiumdioxyd gefällt wird. Säuert man nun eine mit Wollfett verriebene Kaliumosmiatlösung mit verdünnter Schwefelsäure an, so erhält man Osmiumdioxyd in kolloider Form, das von allen organischen Losungsmitteln, die Wollfett lösen, aufgenommen wird.
Präparat 1: Zwei Teile Osmiumtetroxyd werden in acht Teilen 6 prozentiger Natronlauge gelöst und diese Lösung durch wenig Alkohol in der Wärme reduziert. Diese Lösung wird mit 13 Teilen Wollfett verrieben. Hierauf fügt man in kleinen Portionen zehn Teile einer 15 prozentigen Schwefelsäure unter stetigem Verreiben hinzu. Die durch die Natriumosmiatlösung schmutzig violett gefärbte Salbenmause färbt sich blauschwarz. Nach zirka dreistündigem Stehen wird die Mischung durch gelindes Erwärmen erweicht und durch nochmaliges inniges Verreiben die völlige Umsetzung erreicht. Zur Entfernung des Wassers und der Reaktionsnebenprodukte wird das Präparat im Vakuum bei $40-50^{\circ}$ über Schwefelsäure getrocknet und darauf etwa in 100 Teilen Petroläther gelöst; ein Teil der Reaktionsnebenprodukte bleibt unlöslich zurück; die völlige Entfernung von Natriumsulfat geschieht dadurch, daB man die Aetherlösung 2-3 mal mit zirka dem doppelten Volumen Wasser ausschüttelt. Die Petrolätherlösung wird mit Chlorkalzium getrocknet, nach 5-6 Stunden von dem Trockenmittel möglichst abgegossen und der letzte Rest durch Watte filtriert. Destilliert man den Petroläther $\mathbf{a b}$, so resultiert eine schwarz gefärbte salbenartige Masse, die sich. leicht in allen den organischen Lösungsmitteln löst, die Wollfett lösen. Das flüssige Organosol bildete im auffallenden Lichte eine schwarzblaue Flüssigkeit, die im durchfallenden Lichte klar und tiefblau war.

Zur Feststellung des Osmiumgehaltes wurde ein Teil des Präparates direkt im Sauerstoffstrom verbrannt.

Angew. Subst. 2,62 g, gef. Os $=0,213$ Proz. $\mathrm{Os}=8,2=9,53 \mathrm{OsO}_{2}$.

Präparat 2: Um aus derartigen Präparaten solche mit höherem Osmiumdioxyd zu gewinnen, wird ein genau in gleicher Weise hergestelltes Produkt in $70 \mathrm{ccm}$ Petroläther gelöst und aus dieser Lösung durch Zusatz des doppelten Volumens Alkohol das Kolloid gefällt. Der in schwarzblauen Flocken sich abscheidende Niederschlag trennt sich leicht und vollständig von der überstehenden gelb gefärbten Lossung, so dab man diese größtenteils durch vorsichtiges Abgießen beseitigen kann. Der Niederschlag wird auf einem Filter gesammelt, vom Filter noch feucht abgelöst und bei einer Temperatur von $40-50^{\circ}$ getrocknet.

Bei dem Präparat 1 wurde angegeben, daß die Entfernung der Reaktionsnebenprodukte durch Ausschütteln der Petrolätheriösung mit Wasser geschieht. Dabei macht sich jedoch 
oft unangenehm geltend, daB Emulsionen entstehen, die sich nur erst nach recht langer Zeit in Lösung und Wasser scheiden. Bei fast allen Präparaten; in denen der Osmiumgehalt durch Fällen mit Alkohol angereichert wurde, geschah die Reindarstellung dieser in der Weise, daB das durch Alkohol ausgefällte Kolloid möglichst nach Entfernung von Lösungs- und Fällungsmittel wiederhoit mit Wasservon $50-60^{\circ}$ digeriert wurde. Der auf einem Filter gesammelte Niederschlag wurde dann noch so lange mit heiBem Wasser gewaschen, bis in der Wasch- flüssigkeit Schwefelsäure nicht mehr nachweisbar war. Das Präparat wird dann vom Filter abgelöst und in einer Glasschale im Vakuum bei zirka $50^{\circ}$ getrocknet. Das trockene Präparat war schwarzblau, von fester Konsistenz und zeigte sonst die gleichen Eigenschaften wie Präparat 1.

Angew. Subst. 1,35 g, gef. Os 0,283, Proz. Os $20,96=24,5 \mathrm{OsO}_{2}$.

Mitteilung aus der

Königl. Untersuchungsanstalt Erlangen.

\section{Kolloidchemie und Photographie.}

Von Lü ppo-Cramer (Frankfurt a. M.). (Eingagunien an 34. Angust 1916.)

\section{Zur Zerstäubung des Bromsilbers im Lichte.}

In einer früheren Arbeit ") wurde über Versuche berichtet, den von Schultz-Sellack erkannten Antagonismus zwischen der chemischen und der mechanischen Veränderung der Silberhaloide im Lichte auch bei den durch Ammoniak "entwickelten ${ }^{*}$ Bildern zum Ausdruck zu bringen. Es waren hier keine positiven Ergebnisse erzielt und die Mißerfolge dadurch zu erklären versucht worden, daB die Kornoberfläche, die allein man durch chemische Agenzien während der Belichtung beeinflussen kann, im Verhältnis zu der gesamten Masse des Bromsilberkornes von Schichten erheblicher KorngröBe zu gering ist, um bei dem Reifungsprozesse mit Ammoniak einen wirksamen Einflub im Sinne der $S c h u l t z-S e l l a c k$ schen Reaktionen ausüben $z u$ können.

Es wurden deshalb erneut Versuche in jener Richtung mit sogen. kornlosen Bromsilberschichten unternommen, deren Dispersitătsgrad eher mit dem der jodierten Silberspiegel in Vergleich gestellt werden kann. Badet man solche Platten in Bromsalzlösung $(1,5$ prozentige $\mathrm{K} \mathrm{Br}$ Lösung $11 / 2$ Minuten lang) und trocknet, so sieht man sthon ohne weiteres an der gesteigerten Opaleszenz der Schichten, daB eine Reifung infolge der Bromsalzwirkung stattgefunden hat. In der Tat war auch die Empfindlichkeit bei nachfolgender chemischer Entwicklung auf etwa das fünf- bis sechsfache gestiegen, obwohl bei diesen Vergleichen das Bromsalz nicht elnmal vor der Entwicklung ausgewaschen woiden war. Es würde also eine Quelle großer

!) Phot. Korr. 1913, 409.
Fehler bedeuten, wenn man (wie man es bei mittelkörnigen Schichten unbedenklich tun könnte) eine mit Bromsalz imprägnierte Lip p$\mathrm{m}$ a $\mathrm{n}$-Platte mit einer im ursprünglichen $\mathrm{Zu}$ stande befindlichen inbezug auf die Ammoniak. "Entwicklung" vergleichen wolite.

Es wurden daher größere Quantităten von Lippmann-Platten zuerst in der Bromsalzlösung gebadet und getrocknet. Darauf wurde die für die Kontrollplaiten bestimmte eine Halfte des Vorrates ausgewaschen und wiederum unter genau gleichen Verhältnissen wie vorher getrocknet. Die Plattenpaare wurden dann unter geeigneten Schwarzweißnegativen durchschnittlich eine halbe Minute lang vom Sonnenlichte bestrahit. Vor der Entwickiung beobachtet man deutlich, dab die schwache direkt sichtbare Ver:̈nderung auf der mit Bromsalz imprihgnierten Platte erheblich geringer ist als auf der Kontrollplatte. Auch wenn man nach der Belichtung eines Platteupaares unter Jonesskalen auswäscht und mit Metol entwickelt, zeigt sich, daß die in $\mathrm{KBr}$-Gegenwart belichtete Platte um eine ganze Skalenteihe weniger empfindlich is! als die reine Schicht. Legt man aber die Platten auf Petrischalen, die bis fast zum Rande mit Ammoniak $(0,91)$ gefüllt sind, so entstehen zwar die ersten Anfänge des Reifungshildes ziemlich gleichzeitig, aber nach einiger Zeit überholt das Bild auf der bromidhaltigen Platte das andere bedeutend und nach 20 bis $30 \mathrm{Mi}$ nuten stellt sich ein sehr großer Unterschied heraus : es ist ein kontrastreiches Bild entstanden, während es auf der reinen Bromsilberschicht noch recht wenig ausgeprägt ist. Auch wenn man noch die Fehlerquelle ansschaltet, die man darin erblicken könnte, daß während de: 